\title{
Über die toxische Wirkung des Benzols und Gasolins auf die Zellen im lockeren Bindegewebe der Maus."
}

\author{
Von \\ Kikusi Kimura. \\ (Aus dem Anatomischen Institut der Medizinischen Fakultät zu Okayama ; \\ Vorstand: Prof. K. Yagita und Prof. M. Seki.) \\ Mit 19 Textabbildungen. \\ I. Material und Methodik. . . . . . . . . . . . . Is6 \\ II. Experimente mit Benzol. . . . . . . . . . . . . . . . . I57 \\ 1. Inhalation des Benzols. . . . . . . . . . . . I58 \\ a) Zellen des subkutanen Bindegewebes. . . . . . . I58 \\ b) Zellen des subperitonealen Bindegewebes. . . . . . . I60 \\ 2. Injektion des Benzols. . . . . . . . . . . . . . . I6I \\ III. Experimente mit Gasolin. . . . . . . . . . . . . . . . . I63 \\ I. Inhalation des Gasolins. . . . . . . . . . . . . I $\sigma_{3}$ \\ a) Zellen des subkutanen Bindegewebes. . . . . . . . I $\sigma_{3}$ \\ b) Zellen des subperitonealen Bindegewebes. . . . . . $16_{4}$ \\ 2. Injektion des Gasolins. . . . . . . . . . . . . . . . . I64 \\ IV. Zusammenfassung. . . . . . . . . . . . . . I64 \\ Literaturverzeichnis. . . . . . . . . . . . . I6s
}

Schon längst sind Autoren darauf aufmerksam gemacht worden, dass das Benzol im Organismus als leukozytenvermindernd wirkt. Obwohl diesbezügliche zahlreiche Arbeiten veröffentlicht worden sind, so behandeln sie doch hauptsächlich nur Blutbild und pathologisch-anatomische Befunde der blutbildeńden Organen. Unter den Arbeiten ist uns vor allem der Bericht Hetts (1940) interessant, danach erwies sowohl bei der subkutanen Injektion als auch bei der Inhalation des Benzols das Kapillarsystem der Leber sich als Hauptorgan des phagozytären Abbaues, die Milz und das Knochenmark spielten aber bei der Beseitigung der geschädigten Weissen keine Rolle, wobei in der Milz die Vermehrung des lymphoiden Gewebes sich konstatieren liess. Lignac (1932) beschrieb auch eine Vermehrung des lymphoiden Gewebes besonders in der Milz. Nach Miyoshi (1936) ist

r) Diese Arbeit wurde auf Kosten der Ausgaben des Unterrichtsministeriums für wissenschaftliche Forschung ausgeführt. 
die Vermehrung der Lymphozyten auf intensiver Reizung des lymphoiden Gewebes zuzuschreiben.

Was das Knochenmark anbelangt, so sind die darin abspielenden Prozesse in folgender Weise mitgeteilt. Hett beobachtete merkwürdigerweise keine Schädigungen der Stammzellen der Roten und gelapptkerrigen Formen. Carleton (1934) wies darauf hin, dass das retikuloendotheliale System im Knochenmark von Kaninchen durch Benzol wenig beeinflusst wird (nicht aber andere Zellarten). Wallbach (1929) beobachtete bei Benzolinjektion des Kaninchens zahlreiche lymphoide Zellen im ausgepinselten Knochenmark, die in keine Zellklasse unterzubringen sind. Er machte aber darauf aufmerksam, dass unter der Einwirkung eines toxischen Reizes sich abrundende Retikulumzellen dasselbe Aussehen annehmen könnten. Wallbach nahm des weiteren für den Wirkungsmechanismus des Benzols so an, dass nur eine Neubildung und vermehrte Ausreifung von segmentierten granulierten Leukozyten durch bestimmte Hemmung dieser Substanz ausbleibt, dass aber eine vermehrte Zerstörung der weissen Blutkörperchen bei der auch sonst nur beschränkten Lebensdauer der Zellen nicht angenommen zu werden braucht. Über die Beeinflussung des Benzols auf anderweitige Mesenchymapparate, speziell auf die Zellen im lockeren Bindegewebe liegen aber kaum Untersuchungen vor. Watanabe (1933) konnte bei Kaninchen nach der subkutanen Injektion mit Benzol eine Herabsetzung der phagozytischen Funktion der subkutanen histiozytären Zellen beobachten. Unsere nachfolgenden Untersuchungen sollen vor allem versuchen, die nachteilige Beeinflussung des Benzols und Gasolins auf die Umwandlungsfähigkeit der Zellen im lockeren Bindegewebe der Maus zu konstatieren und den Vorgängen näherzukommen.

\section{Material und Methodik.}

Um eine akute Vergiftung durch Benzol hervorzurufen, liess sich eine Maus mit dem Interval von I Stunde 2 mal je $s$ Minuten lang in solchem grossen Exsikator bleiben, in welchem ein mit $0,5 \mathrm{ccm}$ Benzol getränktes Löschpapier eingelegt war, um der Maus Benzoldampf inhalieren zu lassen. Bei der chronischen Vergiftung inhalierte eine Maus Benzol in der oben erwähnten Weișe täglich 2.mal je 3 Minuten lang mit dem Interval von I Stunde. Diese Behandlung wurde 7 Tage lang wiederholt.

Auch der Injektionsversuch wurde in akute und chronische Vergiftung geteilt. Bei jenen wurde $0,15 \mathrm{ccm}$ Lösung von Benzol-Olivenöl ( I : I) nur I mal in den Seitenbauch injiziert, während bei dieser $0,1 \mathrm{ccm}$ BenzolOlivenöl ( 1 : roo) I mal täglich 7 Tage lang injiziert wurde. 
Das Experiment mit Gasolin wurde in gleicher Weise wie das mit Benzol vorgenommen. Für die akute Vergiftung beim Inhalationsversuch liess man die Maus Gasolin je s Minuten 2 mal inhalieren. Für die chronische Vergiftung wurde Gasolin ro mal täglich je 3 Minuten 7 Tage lang wiederholt gegeben. Der Injektionsversuch: Bei der akuten Vergiftung wurde 0, I $5 \mathrm{ccm}$ Gasolin-Olivenöl ( $\mathrm{I}: \mathrm{r}$ ) nur I mal, bei der chronischen Vergiftung o, I ccm I mal täglich 7 Tage wiederholt injiziert.

Die Feststellung des Gewichtes der Tiere, die bei der chronischen Vergiftung alle zwei Tage gemacht wurde, ergab beim Inhalationsexperiment mit Benzol fast keine merkliche Veränderung, dagegen beobachtete man beim Injektionsexperiment nach 7 Tagen eine Verringerung des Körpergewichtes durchschnittlich um 0,9gr. Beim Gasolin scheint die Einwirkung beim Inhalations- und Injektionsexperiment kaum verschieden zu sein.

Die Zellen im lockeren Bindegewebe wurden in folgender Weise untersucht: Nach der Inhalation mit Benzol und Gasolin wurde eine kleine Menge Crotonöl an dem radierten Seitenbauch der Maus bepinselt, und zwar bei der akuten Vergiftung 3, 6, I2, 24 Stunden und 2 und 3 Tage nach der Inhalation und bei der chronischen 3 Stunden nach der letzten Inhalation. 3 Stunden danach wurden Stückchen des subkutanen Bindegewebes dem Seitenbauch des Tieres entnommen. Ferner rieb man Teerpaste, welche die Haut schwächer reizt (Mukohata, I94I b), dem Tiere auf die radierte Haut mit Fingerspitze ein, und 24 Stunden danach wurden Stückchen des Bindegewebes wie oben entnommen.

Beim Injektionsversuche liess sich Crotonöl bzw. Teerpaste 6 Stunden nach der Injektion an die andere Körperșeite applizieren.

Die Bindegewebestückchen wurden auf einen gereinigten Objektträger ausgebreitet und in 10\% iger Formalinlösung fixiert, um nach der v. Möllendorffschen Eisenhämatein-Lackmethode zu färben.

\section{Versuche mit Benzol.}

Vor der Einleitung zur Hauptbesprechung wird das Bild des normalen subkutanen Gewebes (Abb. I) und das der durch die Applikation von Crotonöl und Teerpaste veränderten Zellen angezeigt. Wie schon Mukohata (I94I a u. b) festgestellt hat, wandelt sich ein Teil der Fibrozyten durch das Crotonöl rasch in die Leukozyten um bzw. liefert die letzteren, so dass in 3 Stunden eine grosse Vermehrung der Leukozyten zu beobachten ist (Abb. 2.). Auch durch die Teerpaste verändern sich die Fibrozyten, daraus entstehen in 24 Stunden viel Histiozyten (Abb. 3). 


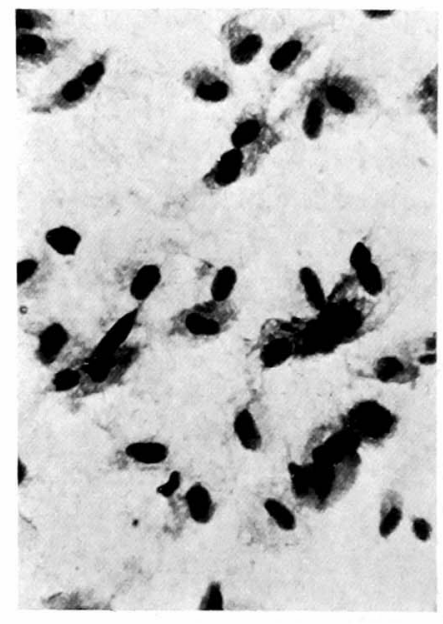

Abb. I. Häutchenpräparat des normalen subkutanen Bindegewebes (Maus). Färbung: Eisenhämatein-Lackmethode. Vergr. $280 \times$.

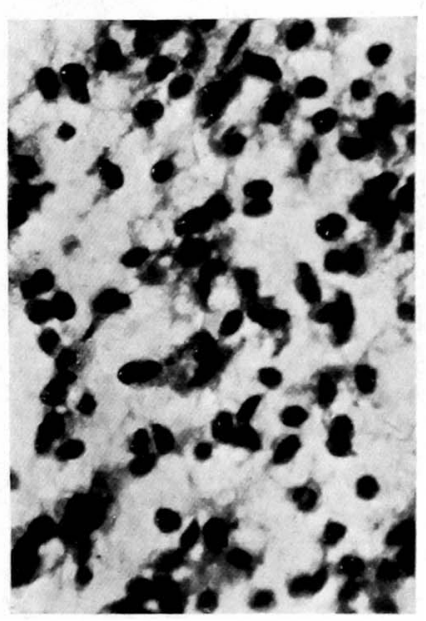

Abb. 2. Subkutanes Bindegewebe, 3 Std. nach Crotonölbepinselung.

Vergr. $280 \times$.

\section{Inhalationsversuch.}

a) Zellen des subkutanen Bindegewebes.

i. Akute Vergiftung.

Nach Ablauf von 3 Stunden akuter Benzolvergiftung wird der Zellkörper der Fibrozyten dünner, verbinden sich durch scharf begrenzte, unregelmässig verästelte Fortsätze miteinander und bilden somit ein deutliches Netzwerk (Abb. 4). Thr etwas kleiner gewordener Kern ist chromatinreich und lässt sich intensiv färben. Auch Fibrohistiozyten und Histiozyten erleiden eine nicht unbeträchtliche Veränderung im Protoplasma, welches Vakuolen von unregelmässiger Grösse in reichlicher Menge enthält und wabig erscheint. Nach Ablauf von 24 Stunden zeigen aber die meisten Zellen die Neigung, zu normalen Fibrozyten zurückzukehren (Abb. s).

Was die Crotonölreaktion angeht, so ergibt sich, dass bei der Aufstreichung des Crotonöls nach 3 Stunden nach der Benzolinhalation Leukozyten merkwürdigerweise nur selten vorkommen. Die Fibrozyten sind noch immer schlank (Abb. 6). Erst nach Ablauf von 6-12 Stunden treten vielerorts Leukozyten und monozytenähnliche Formen auf. Auch Fibrohistiozyten vermehren sich.

Nach Ablauf von 24-48 Stunden nach der Benzolinhalation ziehen die Fibrozyten durch Crotonölapplikation ihre Protoplasmafortsätze mehr oder weniger stark ein und zeigen die Tendenz, rundlich zu werden. Lochkernige 


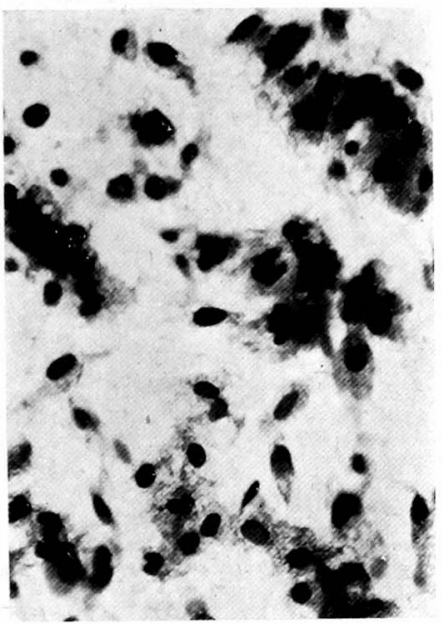

Abb. 3. Subkutanes Bindegewebe, 24 Std. nach Teerpasteeinreibung. Vergr. $280 \times$.

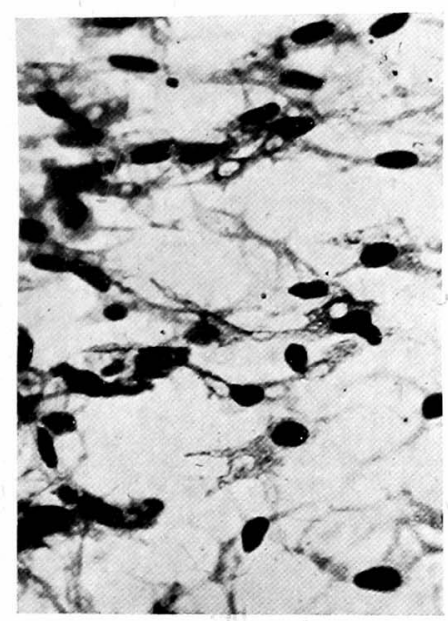

Abb. 5. Subkutanes Bindegewebe, 24 Std. nach Benzolinhalation.

Vergr. 280 $\times$.

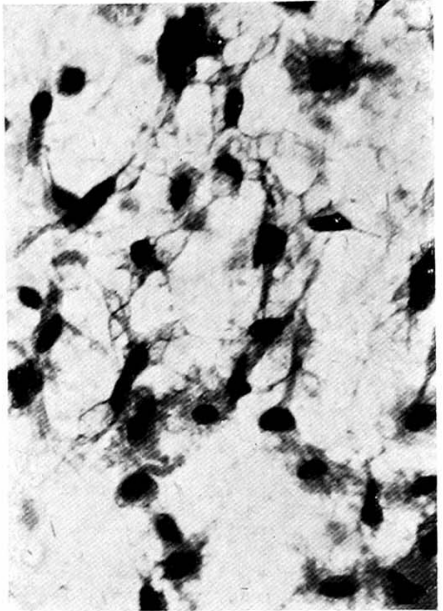

Abb. 4. Subkutanes Bindegewebe, 3 Std. nach Benzolinhalation.

Vergr. 280 X.

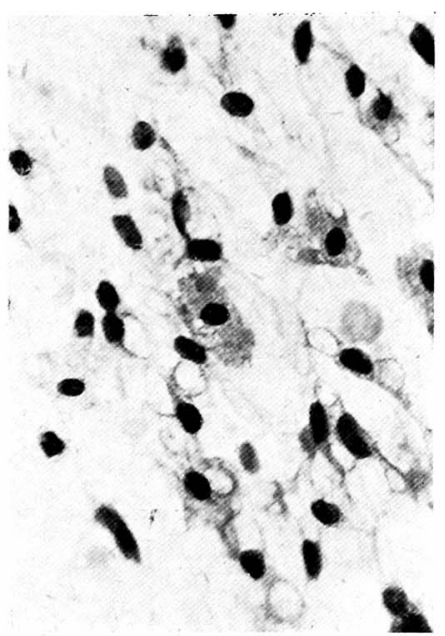

Abb. 6. Subkutanes Bindegewebe Crotonölpinseln (3 Std. nach Benzolinhalation vorgenommen). Vergr. 280X.

Zellen und polymorphkernige Leukozyten nehmen an Zahl zu (Abb. 7). Nach 3 Tagen vermehren sich insbesondere die Leukozyten auf die Crotonölreizung. Fibrohistiozytäre Formen, Histiozyten, monozytäre Formen und Übergange zwischen diesen Zellarten werden nun reichlich angetroffen (Abb. 8).

Nach der Inhalation des Benzols zeigen die Fibrozyten bei Anwendung 


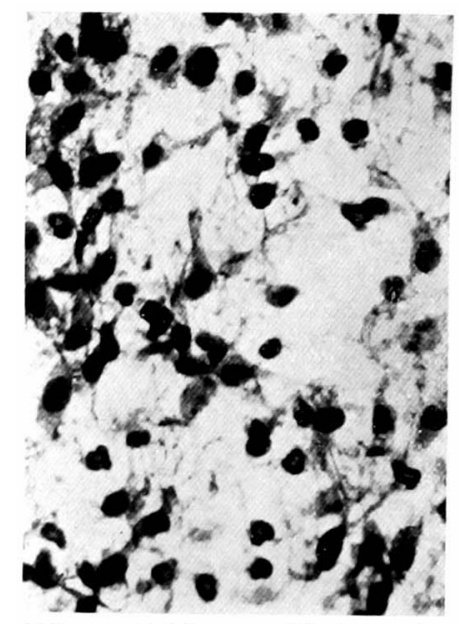

Abb. 7. Subkutanes Bindegewebe. Crotonölpinseln (48 Std. nach Benzolinhalation vorgenommen). Vergr. $280 \times$.

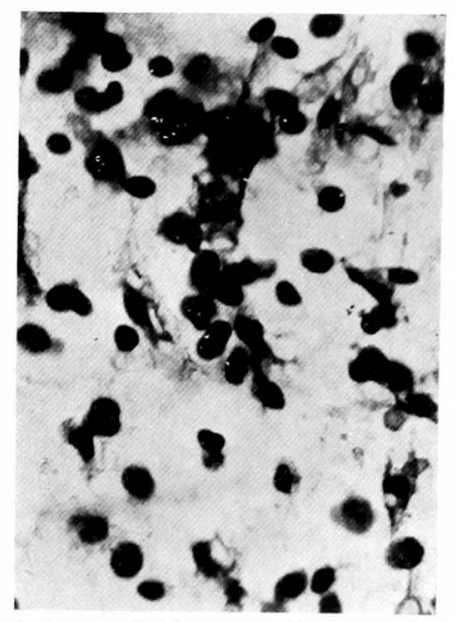

Abb. 8. Subkutanes Bindegewebe.

Crotonölpinseln ( 3 Tage nach Benzolinhalation vorgenommen). Vergr. $280 \times$.

von Teerpaste keine erwähnenswerte Umwandlungen, nur dass sie sich etwas abrunden und oft $\mathrm{I}-2$ grosse Vakuolen in sich einschliessen. Die Kerne lassen sich intensiv färben; ausserdem sind häufig Figuren direkter Kernteilung anzutreffen (Abb. 9).

ii. Chronische Vergiftung.

Bei chronischer Vergiftung an Benzols ist der Zelleib der Fibrozyten gegen die Umgebung scharf abgegrenzt. Die Zellen bilden mit ihren verschmälerten Protoplasmaausläufer ein deutliches Netzwerk (Abb. Io).

Auf Aufstreichung des Crotonöls zeigen die Fibrozyten kaum Umwandlungsfähigkeit (Abb. I I). Fast ebensowenig bei der Teerpasteeinwirkung.

\section{b) Zellen des subperitonealen Bindegewebes.}

Über die Möglichkeit des Entstehens der Leukozyten und Histiozyten aus den Fibrozyten im subperitonealen Gewebe hat Suzue (1937) aufmerksam gemacht. Bei der Maus ist der Befund normaler subperitonealer Bindegewebeszellen etwas anders als der subkutanen (Abb. 12); die Fibrozyten sind in der Regel kleiner, ihr Körper ist schmäler. Fibrohistiozyten und Histiozyten sind vorhanden. 3 Stunden nach der akuten Vergiftung an Benzol werden die Fibrozyten nach unseren genauen Untersuchungen etwas dünner und schliessen vermehrte Vakuolen in sich ein (Abb. I3) $^{\text {) }}$ 


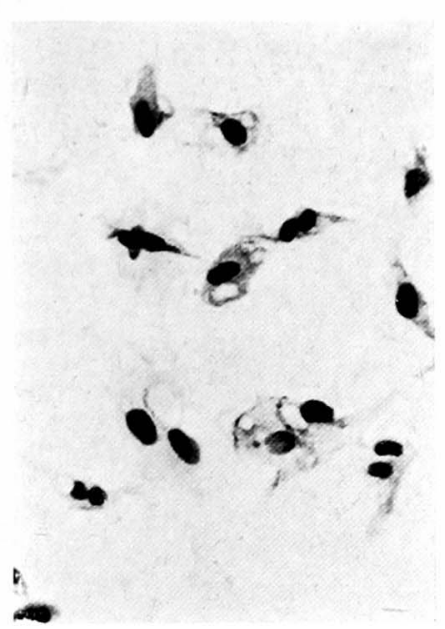

Abb. 9. Subkutanes Bindegewebe. Teerpasteeinreibung (3 Std. nach Benzolinhalation vorgenommen). Vergr. $280 \times$.

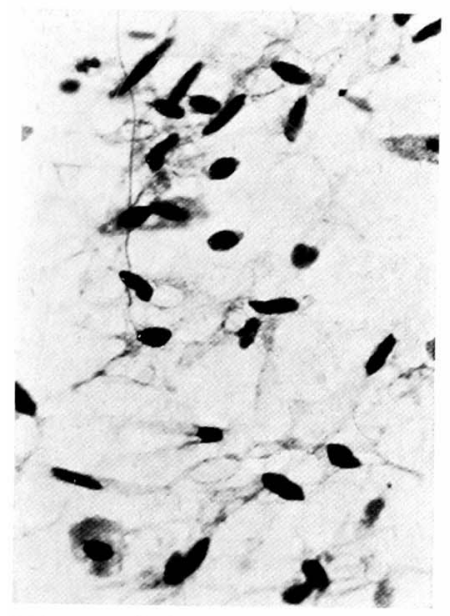

Abb. xI. Subkutanes Bindegewebe. Crotonölpinseln bei chronischer Vergiftung durch Benzolinhalation. Vergr. $280 \times$

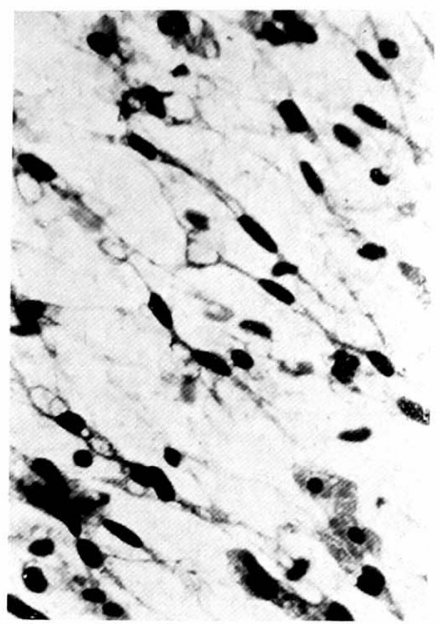

Abb. ro. Subkutanes Bindegewebe, 3 Std. nach einer chronischen, durch Benzolinhalation hervorgerufene Vergiftung. Vergr. $280 \times$.

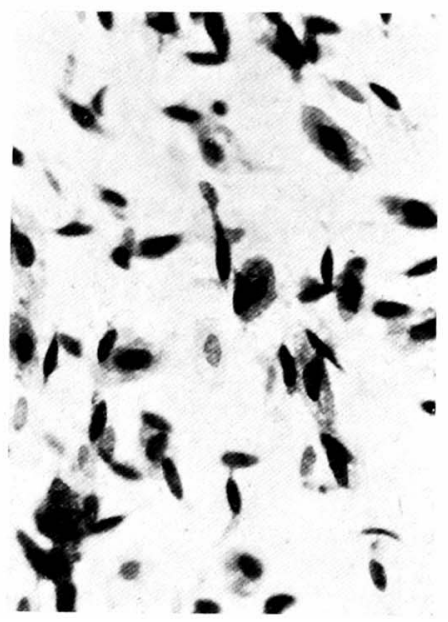

Abb. 12. Normales Subperitonealgewebe (Maus). Vergr. $280 \times$.

\section{Injektionsversuch.}

Es wurde nur das subkutane Gewebe beobachtet.

i. A kute Vergiftung.

In 3 Stunden treten die Veränderungen der Fibrozyten viel beträchtlicher 


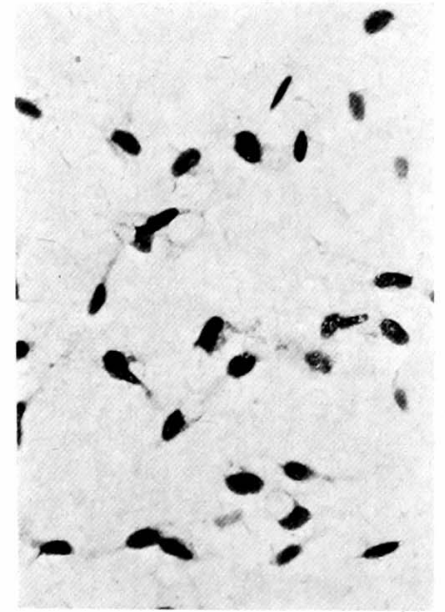

Abb. 13. Subperitoneales Bindegewebe, 3 Std. nach Benzolinhalation. Vergr. $280 \times$.

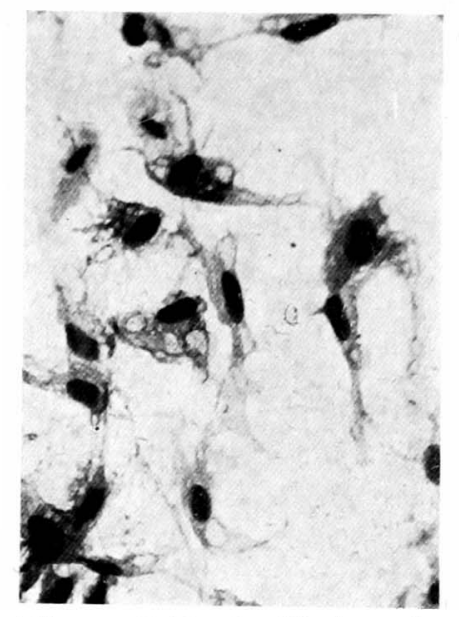

Abb. 15. Subkutanes Bindegewebe, 6 Std. nach Benzolinjektion.

Vergr. $280 \times$.

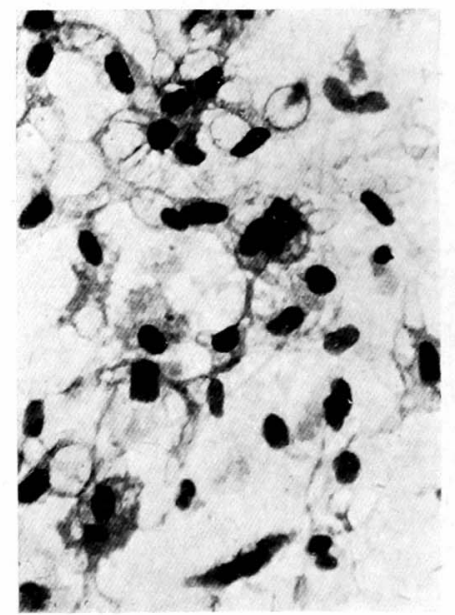

Abb. 14. Subkutanes Bindegewebe, 3 Std. nach Benzolinjektion. Vergr. $280 \times$.

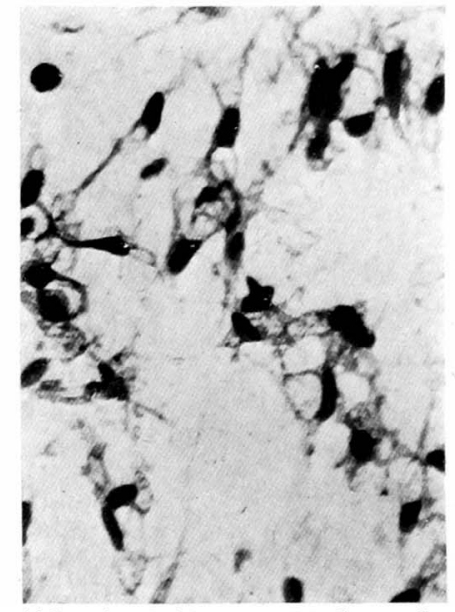

Abb. 16. Subkutanes Bindegewebe.

Crotonölpinseln (6 Std. nach Benzolinjektion vorgenommen). Vergr. $280 X$.

auf als beim Inhalationsversuch; das Protoplasma erleidet eine stärkere Veränderung (Abb. 14). Nach Ablauf von.6 Stunden fangen die Fibrozyten an, zu normalem Zustand zurückzukehren (Abb. I 5 ). 3 Stunden nach der Aufstreichung von Crotonöl ist die Fähigkeit, in Leukozyten überzugehen, hier nicht erkennbar (Abb. I6). Nach der Einreibung von Teerpaste zeigen die Fibrozyten höchstens den Charakter fibrohistiozytärer 


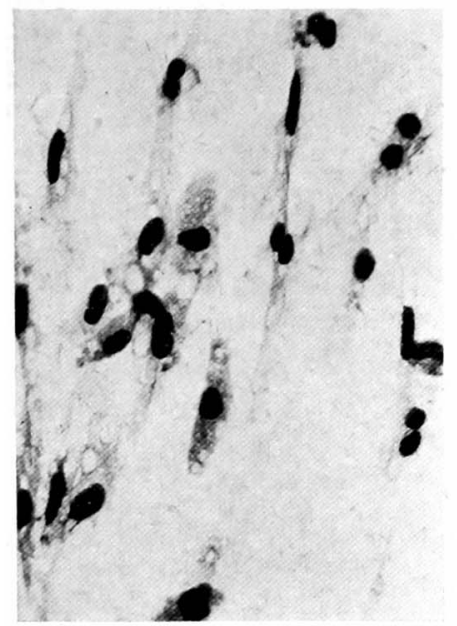
Abb. 17. Subkutanes Bindegewebe. tern begonnen.

Teerpasteeinreibung (6 Std. nach

Benzolinjektion vorgenommen). Vergr. 280 $x$. drückt.
Formen (Abb. i7).

ii. Chronische Vergiftung.

Bei chronischer Vergiftung durch die wiederholten subkutanen Benzolinjektionen sehen die Fibrozyten wie bei akuter Vergiftung aus. Ihre Reaktionsfähigkeit auf die Crotonölaufstreichung ist auch unterge-

Nach der Aufstreichung von Teerpaste runden sich die Fibrozyten etwas ab.

\section{Versuch mit Gasolin.}

Der Versuch wurde jedesmal früh nüch-

\section{Inhalationsversuch.}

a) Zellen des subkutanen Gewebes.

i. Akute Vergiftung.

3 Stunden nach der Vergiftung an Gasolin sind die Veränderungen der Fibrozyten allgemein nicht stark. 3 Stunden nach der Aufstreichung $\mathrm{v}$ on Croton öl treten aber die Leukozyten nur spärlich auf, was uns zeigt, dass die Fibrozyten offenbar beschädigt sind. Die fibrohistiozytären Formen

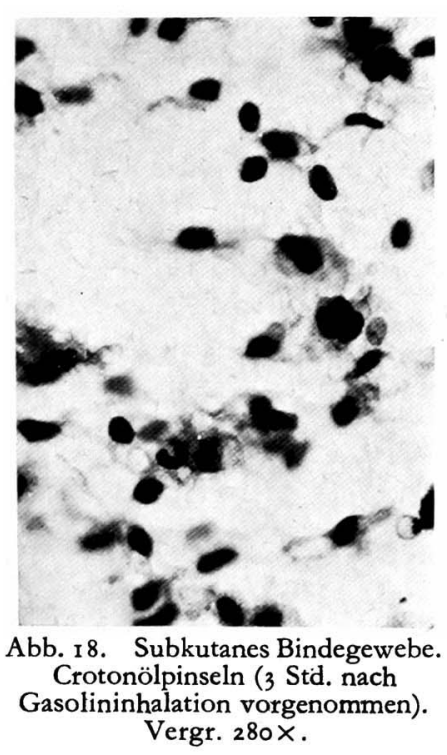

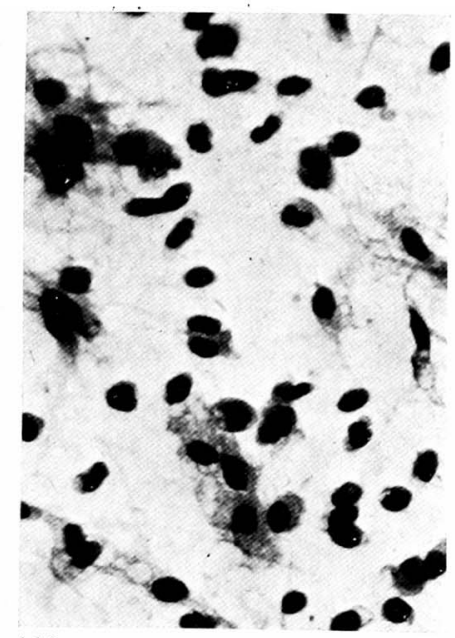

Abb. 19. Subkutanes Bindegewebe. Crotonölpinseln (3 Tage nach Gasolininhalation vorgenommen). Vergr. $280 \times$. 
und Histiozyten können aber sich vermehren (Abb. 18). Nach Ablauf von I bis 3 Tagen gewinnen die Fibrozyten die verlorengegangene Umwandlungsfähigkeit wieder und reagieren auf das Crotonöl mehr oder weniger stark mit Bildung von zahlreichen Leukozyten (Abb. 19). Nach der Teerpasteeinreibung ergeben sich annähernd gleiche Befunde wie bei der akuten Benzolvergiftung durch Inhalation.

ii. Chronische Vergiftung.

Bei chronischer Gasolinvergiftung unter unseren Versuchsbedingungen verändern sich die Zellen im Subkutangewebe nur wenig. Auf Crotonöl und Teerpaste reagieren die Fibrozyten lebhaft.

b) Zellen des subperitonealen Bindegewebes.

Die Zellen erleiden zwar Veränderungen, aber überhaupt viel schwächer als bei der Benzolvergiftung.

\section{Injektionsversuch.}

i. Akute Vergiftung.

3 Stunden nach akuter Gasolinvergiftung werden die Fibrozyten im Subkutangewebe schlanker, die Veränderungen des Protoplasmas treten aber nicht deutlich auf. Nach Ablauf von 6 Stunden kehrt das Aussehen der Fibrozyten wieder zur Norm zurück. Nach Aufstreichung von Crotonöl entstehen fast keine Leukozyten aus den Fibrozyten. Durch die Wirkung von Teerpaste wandelt sich aber ein Teil der Fibrozyten in die fibrohistiozytären Formen.

ii. Chronische Vergiftung.

Die Veränderungen der Fibrozyten sind geringer. Das Crotonöl lässt, wenn auch in kleiner Zahl, fibrohistiozytäre Formen, Histiozyten und Leukozyten aus den Fibrozyten entstehen. Nach Anwendung der Teerpaste werden fibrohistiozytäre Formen, und Histiozyten ziemlich viel beobachtet. Alles im allen wirkt das Gasolin, Gemisch von Kohlenwasserstoffen mit einer offenen Kette, im gleichen Sinne wie das zyklische Benzol, aber in viel schwächerem Grade.

\section{Zusammenfassung.}

I. Bei akuter V'ergiftung sowohl durch die Inhalation als auch durch die Injektion von Benzol und Gasolin werden bei Mäusen die meisten Fibrozyten, Fibrohistiozyten und Histiozyten des subkutanen Bindegewebes 
erheblich dünner, schliessen aber vermehrte und vergrösserte Vakuolen in sich ein. Das Aussehen der Zellen kehrt nach Ablauf von 6 Stunden schon bei weitem und von 24 Stunden fast vollständig wieder zur Norm zurück. Im subperitonealen Bindegewebe werden auch die Fibrozyten und Histiozyten in beträchtlichem Masse geschädigt. Bei chronischer Vergiftung wurden jedoch die Zellen bei unseren Versuchsbedingungen allgemein nicht so stark geschädigt.

2. Wenn man auf die Haut unbehandelter Mäuse Crotonöl einwirken lässt, wird ein Teil der Fibrozyten, nachdem sie ihre Protoplasmafortsätze einzieht, zu Monozyten und Leukozyten. Die Fibrozyten werden aber, wenn man Teerpaste wirken lässt, zu Histiozyten. Bei den Mäusen, denen Benzol oder Gasolin durch Inhalation oder Injektion beigebracht wurde, sieht man, dass die meisten Zellen auf das Crotonöl und die Teerpaste merkwürdigerweise nicht lebhaft reagieren und immer schlank bleiben. Die Umwandlungsfähigkeit der Fibrozyten stellte sich aber nach Ablauf von Zeiten ( $1-3$ Tagen) wieder auf die Norm ein.

\section{Literaturverzeichnis.}

Carleton, H. M.: Studies on epitbelial phagocytosis. II. A method of demonstrating the origin of dust cells. Proc. Roy. Soc., B, 144 (1934).

Hett, J.: Über den Leukozytenabbau im tierischen Körper. Experimentelle Untersuchungen an Benzolmäusen. Z. Zellforsch., 30 (1940).

Lignac, G. O. E.: Die Benzolleukämie bei Menschen und weissen Mäusen. Krankheitsforsch., 9 (1932).

Miyoshi, T.: Befunde des Blutes und der hämatogenen Organe der Maus bei chronischer Benzolvergiftung. Juzenkai Z., 4I (1936). (Jap.)

Mukohata, J.: Experimentelle Untersuchungen über die Umwandlungsfähigkeit der Zellen im Subkutangewebe der Maus. III. Versuche mit Crotonöl. Okajimas Fol. anat. jap., 20 (I94I a).

- - : Experimentelle Untersuchungen über die Umwandlungsfähigkeit der Zellen im Subkutangewebe der Maus. IV. Versuche mit Teerpaste von Dohi. Okajimas Fol. anat. jap. 20 (x94I b).

Suzuye, Y.: Ubber die bindegewebigen Zellen im Subperitonealgewebe. I. Untersuchungen am normalen Tiere. Fol. anat. jap. Is (1937).

_- U U Uber die bindegewebigen Zellen im Subperitonealgewebe. II. Experimentelle Untersuchungen über die Veränderungen der Zellen. Fol. anat. jap., 15 (1937).

Wallbach, G.: Untersuchungen über die unterschiedliche Wirkung einiger leukozytenvermindernden Substanzen. Z. exper. Med. 68 (1929).

Watanabe, S.: Über die Veränderung der Phagozytose der subkutanen Histiozyten bei verschiedenen experimentellen Erkrankungen. II. Benzolvergiftung. Juzenkai Z., 38 (r933). (Jap.) 